\title{
Case Series of Morbihan Disease (Extreme Eyelid Oedema Associated with Rosacea): Diagnostic and Therapeutic Approaches
}

\author{
Jung Eun Kim, Chang Yoon Sim, A Young Park, Soon Auck Hong ${ }^{1}$, Young Lip Park², \\ Sun Young Jang ${ }^{3}$, Sung Yul Lee \\ Departments of Dermatology and ${ }^{1}$ Pathology, Soonchunhyang University College of Medicine, Cheonan, Departments of ${ }^{2}$ Dermatology and \\ ${ }^{3}$ Ophthalmology, Soonchunhyang University College of Medicine, Bucheon, Korea
}

Morbihan disease (MD) is a rare form of rosacea that presents with chronic erythema and solid oedema on the upper half of the face. A diagnosis of MD can be made only after eliminating diseases that are similar in terms of clinical and histopathological presentation. The cause of MD remains unclear and no standardised treatment is yet available. MD often tends to be recalcitrant to therapies commonly used to treat rosacea, including systemic corticosteroids, antibiotics, isotretinoin, and topical regimens. Thus, surgical interventions have been attempted but most cases have exhibited unsatisfactory responses. We treated six patients with extreme eyelid lymphoedema without any other cutaneous manifestation. Surgical eyelid reduction was performed in all patients, because ptosis and narrowing of the visual field were the major complaints. Histopathological tests revealed various extents of perivascular and perifollicular inflammation, and dermal oedema. After surgery, patients with severe inflammatory cell infiltration (including mast cells) exhibited a tendency toward recurrence. Other patients with severe dermal oedema exhibited better responses to surgical reduc-

Received August 4, 2017, Revised November 27, 2017, Accepted for publication December 11, 2017

Corresponding author: Sung Yul Lee, Department of Dermatology, Soonchunhyang University College of Medicine, 31 Suncheonhyang 6-gil, Dongnam-gu, Cheonan 31151, Korea. Tel: 82-41-570-2270, Fax: 82-41570-2283, E-mail: dermsung@schmc.ac.kr ORCID: https://orcid.org/0000-0002-6995-4561

This is an Open Access article distributed under the terms of the Creative Commons Attribution Non-Commercial License (http://creativecommons. org/licenses/by-nc/4.0) which permits unrestricted non-commercial use, distribution, and reproduction in any medium, provided the original work is properly cited.

Copyright $₫$ The Korean Dermatological Association and The Korean Society for Investigative Dermatology tion, and thus no recurrence. We propose that MD should be included in the differential diagnosis of persistent, chronic eyelid oedema even if eyelid oedema is the only manifestation; the histological features may aid in the selection of appropriate therapeutic strategies. We suggest that eyelid reduction surgery can be a useful treatment option for MD patients when there is no massive mast cell infiltration. (Ann Dermatol 31(2) 196 200, 2019)

\section{-Keywords-}

Edema, Eyelids, Rosacea

\section{INTRODUCTION}

Morbihan disease (MD) is a rare form of rosacea that presents with chronic erythema and solid oedema on the upper half of the face. A diagnosis of MD can be made only after eliminating diseases that are similar in terms of clinical and histopathological presentation. The cause of MD remains unclear and no standardized treatment is yet available. MD often tends to be recalcitrant to therapies commonly used to treat rosacea, including systemic corticosteroids, antibiotics, isotretinoin, and topical regimens. Thus, surgical interventions have been attempted but most cases have exhibited unsatisfactory responses. We treated six patients with extreme eyelid lymphoedema without any other cutaneous manifestation. Surgical eyelid reduction was performed in all patients, because ptosis and narrowing of the visual field were the major complaints. Histopathological tests revealed various extents of perivascular and perifollicular inflammation, and dermal oedema. After surgery, patients with severe inflammatory 
cell infiltration (including mast cells) exhibited a tendency toward recurrence. Other patients with severe dermal oedema exhibited better responses to surgical reduction, and thus no recurrence. We propose that MD should be included in the differential diagnosis of persistent, chronic eyelid oedema even if eyelid oedema is the only manifestation; the histological features may aid in the selection of appropriate therapeutic strategies. We suggest that eyelid reduction surgery can be a useful treatment option for MD patients when there is no massive mast cell infiltration.

MD, also known as rosacea lymphoedema, is a rare entity characterized by persistent lymphoedema of the upper half of the face ${ }^{1}$. Although an association with rosacea has been suggested, the underlying pathogenic mechanism remains unclear. We treated six patients with extensive non-pitting eyelid lymphoedema and without any other symptom of rosacea. Herein, we describe their notable clinical features, histopathological findings, and treatment outcomes.

\section{CASE REPORT}

We retrospectively studied six patients who presented in 2015 and 2016 with eyelid MD; all exhibited persistent, extensive eyelid oedema requiring surgical eyelid reduction. We received the patient's consent form about publishing all photographic materials. Their clinical details, including age and sex, symptoms, disease duration, any previous history of rosacea or any other dermatosis, histopathological findings, and treatment outcomes are summarized in Table 1.

Our case series consisted of six Koreans aged 42 to 73 years. Disease duration ranged from 8 months to 3 years. The eyelids (both) were the only areas involved in all patients, none of whom had any previous history of rosacea or any other dermatosis. Laboratory data (including antinuclear antibody levels) were normal in all six patients. Head computed tomographic data were also normal except for diffuse infiltration into both eyelids. All patients complained of persistent eyelid oedema, accompanied by narrowing of the visual field; they underwent surgical eyelid reduction followed by lymphatic drainage (Fig. 1A C). Diagnoses were based on characteristic clinical features and histopathological data obtained from the surgically excised specimens. All specimens were stained with hematoxylin and eosin, and tagged antibodies against CD68 and CD117. Histopathologically, all patients exhibited dermal oedema with perivascular and perifollicular inflammatory cell infiltration (Fig. 2). However, the histological features were of two different types. Three patients exhibited severe perivascular and perifollicular inflammatory

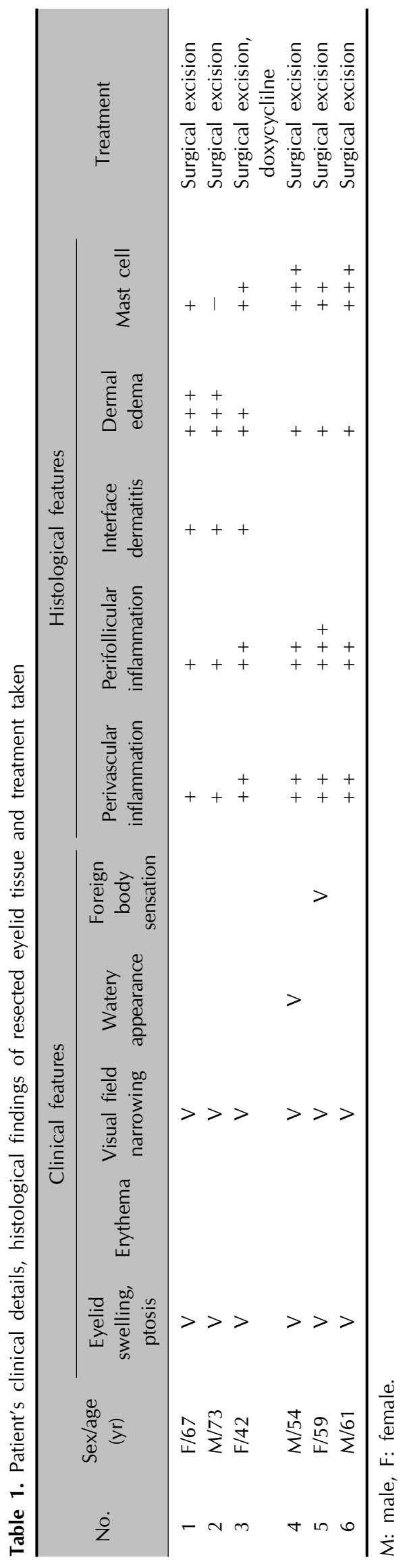

Vol. 31, No. 2, 2019 

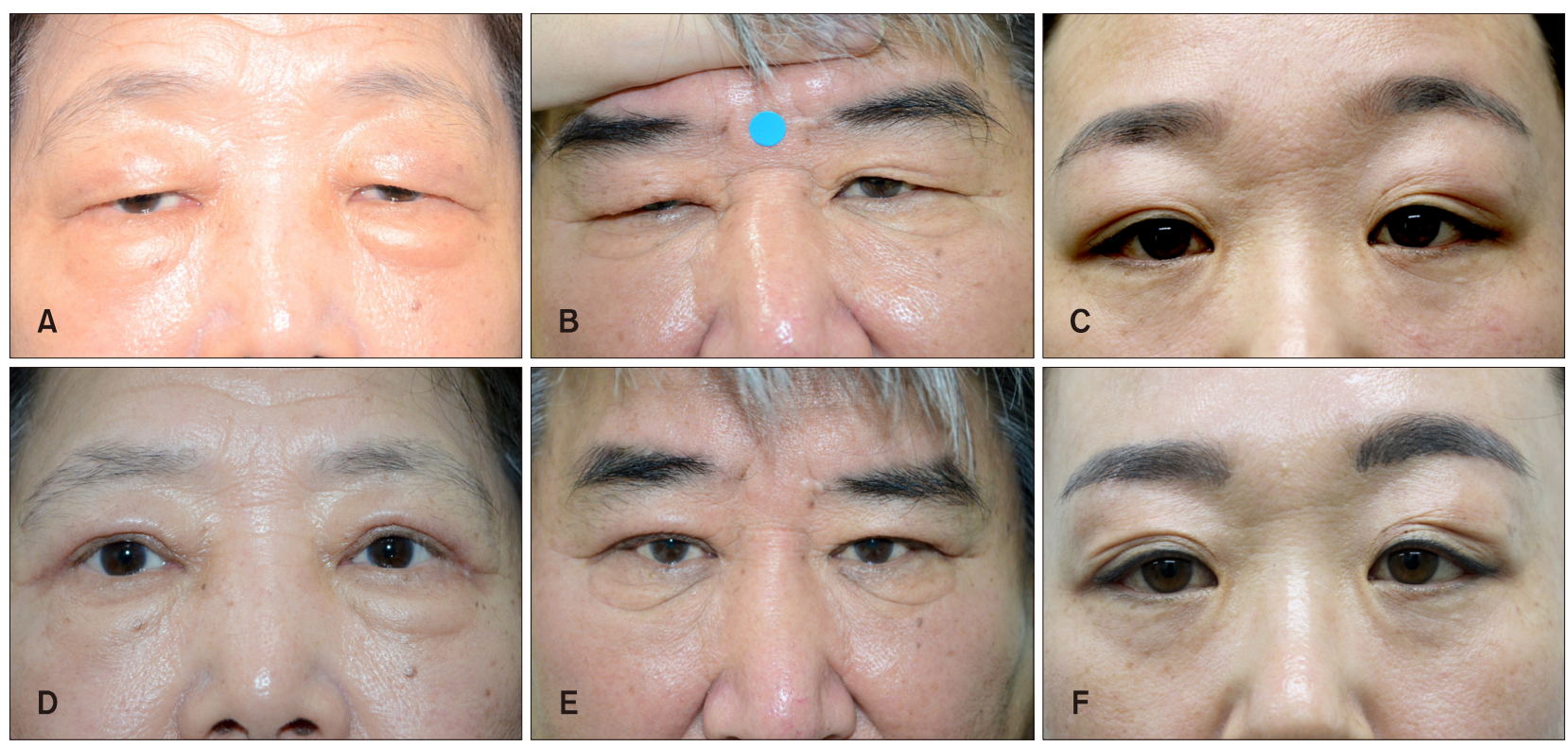

Fig. 1. Initial clinical presentation of patient 1 to 3 . (A $\sim$ C) Soft, non-pitting oedema without erythema on both eyelids. Clinical presentation after 6 months of surgical reduction of patient 1 to 3. (D F) All showed marked improvement.
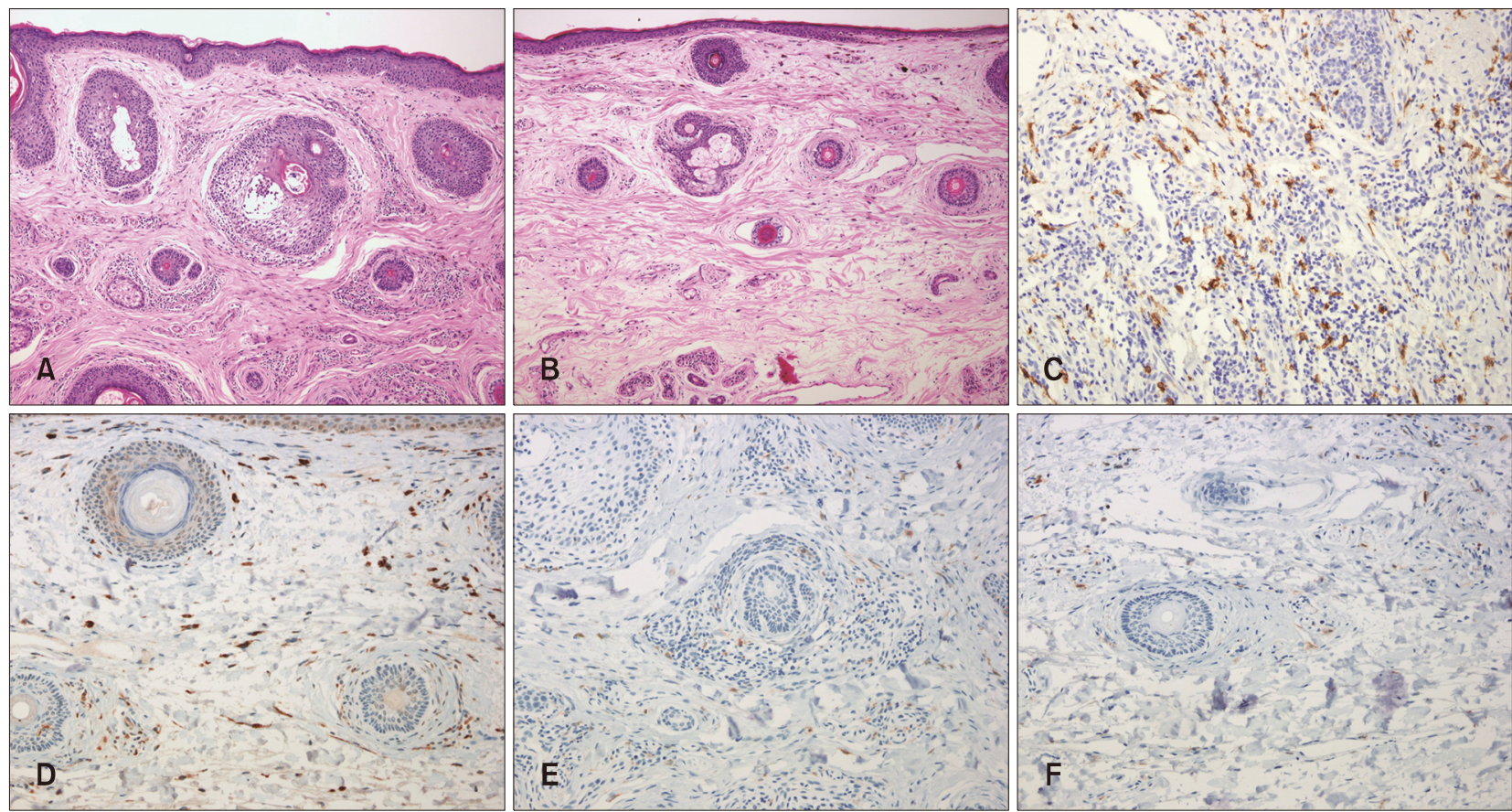

Fig. 2. On H\&E staining, (A) perifollicular inflammation with minimal oedema of dermis in inflammatory type and (B) mild perifollicular inflammation in oedema type are found $(\mathrm{H} \& \mathrm{E}, \times 100)$. (C) Immunohistochemistry $(\mathrm{IHC})$ for $\mathrm{CD} 117$ shows the increased number of mast cell in inflammatory group, (D) comparing to those of odedma group (IHC, $\times 200$ ). (E) The number of CD68-positive histiocyte is increased in inflammatory group than in $(F)$ odema group $(\mathrm{IHC}, \times 200)$.

cell infiltration, including of lymphohistiocytes and many mast cells. In contrast, severe dermal oedema, interface dermatitis, and sun damage were the major findings in two patients. The final patient exhibited mixed histological features with moderate dermal oedema and moderateto-severe inflammatory lymphohistiocytic infiltration with abundant mast cells.

All six patients underwent surgical reduction of the eyelids 
and levator muscles followed by lymphatic drainage. All showed marked improvement (Fig. 1D F). Two patients (no. 1 and 2) did not experience any recurrence at that time, and were satisfied with their results. However in three cases (no. 4 6), recurrence developed by the 6-month follow-up. One patient (no. 3) felt that surgery had afforded significant improvement, but developed slow intermittent swelling of both eyelids, although the symptoms were much less bothersome than previously. We commenced oral doxycycline at $200 \mathrm{mg} /$ day; the symptoms were gradually relieved and observation is continuing. Interestingly, the three patients who experienced recurrences were of the histological type associated with severe perivascular and perifollicular inflammatory cell infiltration, including of lymphohistiocytes and many mast cells. The two patients who did not experience recurrence were of the other histological type, characterized by severe dermal oedema and sun damage. The patient who developed intermittent eyelid swelling after surgery had mixed histological features.

\section{DISCUSSION}

MD was first reported by Degos et al. ${ }^{2}$ in 1957. Clinically, the manifestations usually include firm, non-pitting oedema and erythema, particularly of the glabella, eyelids, forehead, nose, and cheeks ${ }^{1}$. Patients often have no subjective symptoms (itching or pain), or only mild symptoms, but eyelid lymphoedema is commonly accompanied by narrowing of the visual field and ptosis ${ }^{3}$. In severe cases, the ptosis can be almost complete. Although $M D$ is occasionally regarded as a rare complication of chronic rosacea associated with ocular involvement, MD can occur without any symptoms of rosacea ${ }^{4}$. Thus, it is unclear whether MD should be considered as a standalone condition, or a complication caused by rosacea ${ }^{1,3,5}$. No histopathological study on MD has yet appeared. Generally, histopathological findings include dermal oedema, vascular hyperplasia and dilatation, perifollicular fibrosis, and perivascular and perifollicular inflammatory cell infiltration (lymphocytes, with occasional neutrophils, eosinophils, mast cells, and plasma cells ${ }^{1,4}$. However, MD has no known pathognomonic histological feature, and diagnosis thus remains difficult, usually being made after exclusion of various conditions that are similar in appearance, such as dermatomyositis, systemic lupus erythematosus, and chronic actinic dermatitis ${ }^{3}$.

All of our patients presented with extreme eyelid oedema but no other lesion, and they all considered the ptosis and visual field narrowing to be major problems; we thus performed eyelid reduction surgery. The clinical manifesta- tions were very similar among the patients, so we could not predict the histological differences between them. However, histopathological examination showed that the MD was of two different histological types, one of which was associated with severe perivascular and perifollicular inflammation with abundant mast cells; and the other with severe dermal oedema, interface dermatitis, and sun damage. One patient had a mixed histological pattern with both dermal oedema and inflammation. The treatment outcomes differed somewhat by histological type. The inflammationpredominant MD was associated with recurrence but the dermal oedema-predominant type was not.

The pathogenesis of MD remains unknown. Nagasaka et al. ${ }^{1}$ suggested that closure of lymphatic vessels caused by granulomatous infiltration might explain the oedema. Jansen and Plewig ${ }^{6}$ observed infiltration of mast cells around lymphatic vessels, and suggested that such cells might play an important role in disease development, although mast cell infiltration is not a consistent feature of $\mathrm{MD}^{1}$. Mast cells have been shown to be upregulated in the rosacea lesion. Recently, Mascarenhas et al. ${ }^{7}$ suggested that TRPV4 played an important role in LL-37-driven mast cell activation. Although the wider pathological implications are still not clearly understood, mast cells may partly explain the pathogenesis and clinical diversity of rosacea. In addition, Aroni et al. ${ }^{8}$ suggested that mast cell numbers were associated with disease duration. Mast cells were thought to contribute significantly to lesional persistence, and functional endothelial compromise. Thus, we assumed that these features might trigger specific forms of rosacea such as MD.

Treatment of MD is often difficult; no standard regimen has yet emerged. Systemic corticosteroids, e.g. tetracycline, thalidomide, and isotretinoin are often used, but most cases exhibit a chronic refractory course and inadequate response ${ }^{9}$. Surgery is also possible, but recurrence is common ${ }^{10}$. MD associated with massive mast cell infiltration has been treated with long-term oral antibiotics ${ }^{5,11,12}$. Fujimoto et al. ${ }^{11}$ suggested that the extent of response to tetracycline was associated with the degree of mast cell infiltration. Further study of the role played by mast cells in pathogenesis and prognosis is required; the fact that the disease responds to tetracycline does indeed suggest that mast cells play a particular role. We noted no recurrence in patients whose principal histological feature was dermal oedema. However, patients with abundant mast cell infiltration experienced recurrence. The degree of inflammation may be partially related to treatment outcomes, but we thought it important to explore the influence of mast cell infiltration.

Shim et al. ${ }^{13}$ reported a case of $M D$ in Korea, the patient 
was successfully treated with doxycycline and colchicine over 7 months. No recurrence was detected after 14 months of follow-up. To the best of our knowledge, this is the only reported case of MD to date in Korea.

In summary, we report six MD cases in whom extreme eyelid oedema was the only symptom. We observed two histologically different patterns that seemed to affect the treatment outcome after identical surgery. We propose that MD should be included in the differential diagnosis of persistent, chronic eyelid oedema; the histological features may aid in the choice of an appropriate therapeutic strategy. Eyelid reduction surgery may be a useful treatment option for MD without massive mast cell infiltration.

\section{ACKNOWLEDGMENT}

This study was supported by the National Research Foundation of Korea (NRF-2017R1A1A1A05001051) and the Soonchunhyang University Research Fund.

\section{CONFLICTS OF INTEREST}

The authors have nothing to disclose.

\section{ORCID}

Jung Eun Kim, https://orcid.org/0000-0002-8399-8456

Chang Yoon Sim, https://orcid.org/0000-0003-4843-664X

A Young Park, https://orcid.org/0000-0002-9775-0654

Soon Auck Hong, https://orcid.org/0000-0002-7902-4608

Young Lip Park, https://orcid.org/0000-0002-6532-3156

Sun Young Jang, https://orcid.org/0000-0002-0613-8356

Sung Yul Lee, https://orcid.org/0000-0002-6995-4561

\section{REFERENCES}

1. Nagasaka T, Koyama T, Matsumura K, Chen KR. Persistent lymphoedema in Morbihan disease: formation of perilym- phatic epithelioid cell granulomas as a possible pathogenesis. Clin Exp Dermatol 2008;33:764-767.

2. Degos R, Civatte J, Beuve-Méry M. Nouveau cas d'oedème érythémateux facial chronique. Bull Soc Fr Dermatol Syph 1973;80:257.

3. Wohlrab J, Lueftl M, Marsch WC. Persistent erythema and edema of the midthird and upper aspect of the face (morbus morbihan): evidence of hidden immunologic contact urticaria and impaired lymphatic drainage. J Am Acad Dermatol 2005;52:595-602.

4. Lai TF, Leibovitch I, James C, Huilgol SC, Selva D. Rosacea lymphoedema of the eyelid. Acta Ophthalmol Scand 2004; 82:765-767.

5. Okubo A, Takahashi K, Akasaka T, Amano H. Four cases of Morbihan disease successfully treated with doxycycline. J Dermatol 2017;44:713-716.

6. Jansen T, Plewig G. The treatment of rosaceous lymphoedema. Clin Exp Dermatol 1997;22:57.

7. Mascarenhas NL, Wang Z, Chang YL, Di Nardo A. TRPV4 mediates mast cell activation in cathelicidin-induced rosacea inflammation. J Invest Dermatol 2017;137:972-975.

8. Aroni K, Tsagroni E, Kavantzas N, Patsouris E, loannidis E. A study of the pathogenesis of rosacea: how angiogenesis and mast cells may participate in a complex multifactorial process. Arch Dermatol Res 2008;300:125-131.

9. Crawford GH, Pelle MT, James WD. Rosacea: I. Etiology, pathogenesis, and subtype classification. J Am Acad Dermatol 2004; 51:327-341; quiz 342-344.

10. Bernardini FP, Kersten RC, Khouri LM, Moin M, Kulwin DR, Mutasim DF. Chronic eyelid lymphedema and acne rosacea. Report of two cases. Ophthalmology 2000;107:22202223.

11. Fujimoto N, Mitsuru M, Tanaka T. Successful treatment of Morbihan disease with long-term minocycline and its association with mast cell infiltration. Acta Derm Venereol 2015; 95:368-369.

12. Kabuto M, Fujimoto $N$, Honda S, Tanaka T. Successful treatment with long-term use of minocycline for Morbihan disease showing mast cell infiltration: a second case report. J Dermatol 2015;42:827-828.

13. Shim WH, Jwa SW, Song M, Kim HS, Ko HC, Kim BS, et al. Morbihan disease: a rare variant of rosacea. Korean J Dermatol 2012;50:320-323. 